\title{
Real-time detection and control of microchannel resonance frequency in acoustic trapping systems by monitoring amplifier supply currents
}

\author{
Vahid Farmehini ${ }^{a, \#}$, Sadie Kiendzior ${ }^{b, \#}$, James P. Landers ${ }^{b}$, and Nathan S. Swami*a,b \\ a - Electrical and Computer Engineering, University of Virginia, Charlottesville, Virginia 22904, USA; \\ b - Chemistry, University of Virginia, Charlottesville, Virginia 22904, USA; \\ \# Authors contributed equally
}

\section{Supplementary Information}

Section \# \& Subtitle

Page

S1. Comparison of Peak Detector Approaches

2

S2. Sensitivity Analysis Calculation

4

S3. AC Current Measurement circuit - an example

5

S4. Current-meter unit

6

S5. Details on Resonance Frequency Tracking

6

S6. Multiplexed Acoustic Trapping system

8

Captions for the Uploaded Video *.avi files:

Fig.4_CurrentMode.avi: Current mode operation of Figure 4 of the main manuscript document

Fig.4_VoltageMode.avi: Voltage mode operation of Figure 4 of the main manuscript document

Fig.5_CurrentMode.avi: Current mode operation of Figure 5 of the main manuscript document

Fig.5_VoltageMode.avi: Voltage mode operation of Figure 5 of the main manuscript document Fig.6_CurrentMode.avi: Current mode operation of Figure 6 of the main manuscript document Fig.6_VoltageMode.avi: Voltage mode operation of Figure 6 of the main manuscript document

* Corresponding Author. Fax: +1-434-924-8818.

Email: nswami@virginia.edu 


\section{S1. Comparison of Peak Detector Approaches}

Fig. S1a presents the schematics of an improved design for the classic Peak Detector. The accuracy of the circuits is relatively flat (error $-300 \mathrm{mV}$ ) for low input amplitude throughout the entire frequency range (1-10 MHz). However, the error will be more significant for larger inputs as the frequency increases beyond $7 \mathrm{MHz}$, where our acoustic Trapping experiments need. Fig. S1b shows the variation of absolute error $(\mathrm{mV})$ in the megahertz range for several input amplitudes. The "absolute error" is the difference between DC output (OUT) and input amplitude (IN) of the Peak detector. We have employed a fast OPAmp (LM6171, Texas Instrument, TX) with a slew rate of $3600 \mathrm{~V} / \mu \mathrm{s}$.

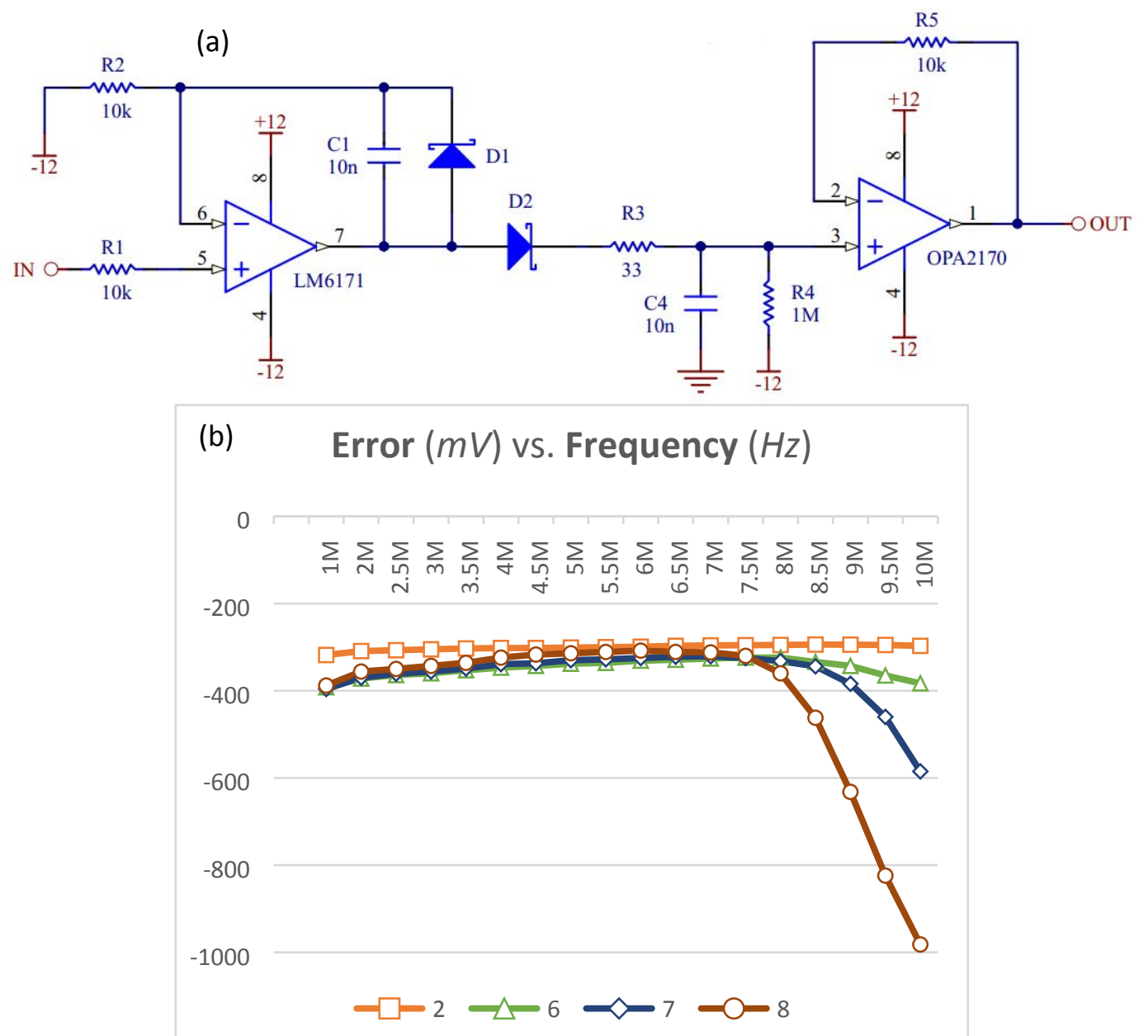

Fig. S1 (a) The schematic of Improved Classic Peak detector (b) The absolute error (mV) over 1 to $10 \mathrm{MHz}$ frequency range, measured for four different input amplitudes in volt (Peak-Peak).

Fig. S2a presents another novel design for peak detection of a high-frequency signal. This design uses a high-speed comparator (AD8561, Analog Devices, MA) with a propagation delay of 7 nanoseconds and three sub-amplifiers of OPA4170 (Texas Instruments, TX) that serve as a buffer. One key feature of this design is that the charging current into the Charge holder capacitor (C3) is independent of the capacitor voltage ( $\mathrm{Vc} 3$ ), due to the boosting current provided by buffer \#2. Without this injected boosting current, the charging current becomes very small as the capacitor voltage reaches the target voltage (Input 
amplitude). This reduced current in higher frequencies may be unable to charge the capacitor sufficiently. Therefore, absolute error may increase accordingly.
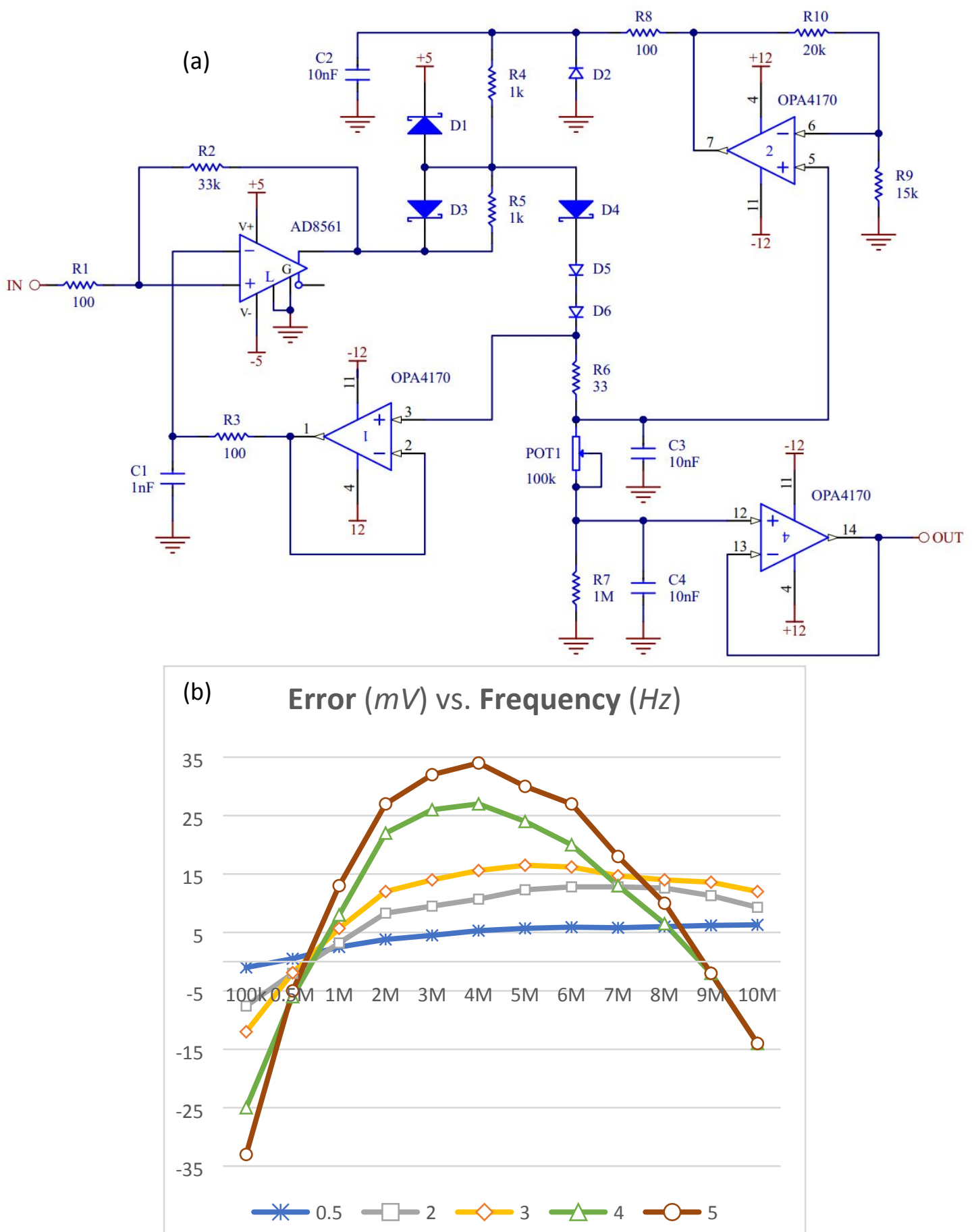

Fig. S2 (a) The schematics of Wideband Comparator-based Peak detector with Current booster. (b) The absolute error (mV) over 1 to $10 \mathrm{MHz}$ frequency range, measured for five different input amplitudes in volt (Peak-Peak). 


\section{S2. Sensitivity Analysis Calculation}

We can model a non-ideal amplifier as a combination of an ideal one $\left(V_{\text {out }}=V s\right)$ with an output resistance $\left(R_{o}\right)$ as shown in Fig. $\mathbf{S 3}$. We can easily calculate the amplitude of AC signal across piezo $\left(V_{p}\right)$ by using the voltage division between $R_{o}$ and $Z_{\text {Piezo }}$ :

$$
V_{p}=V_{s}\left(\frac{\left|Z_{\text {Piezo }}\right|}{\left|Z_{\text {Piezo }}+R_{o}\right|}\right)
$$

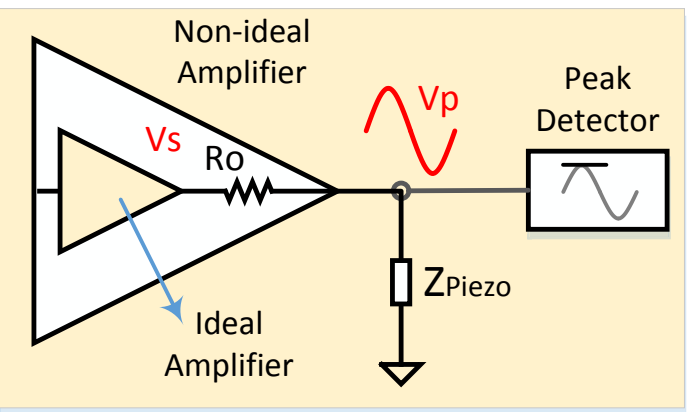

Fig. S3 A non-ideal amplifier $(R o \neq 0)$ that stimulates a piezoelectric transducer with AC signal $V p$

As the frequency approaches the resonance frequency, the impedance becomes mostly resistive in its minimum magnitude $(Z p)$. Hence, the amplitude $V p$ will reach a minimum as well, according to Eq. (1).

The amount of current drawn from the amplifier makes the voltage drop across Ro maximized at the resonance frequency. Hence, by comparing these voltage drops in different frequency points and finding the local minimum of voltage amplitude across the piezo, we can determine the optimum frequency of trapping.

\section{S2.1. Voltage sensitivity}

By taking the differential from both sides of Eq. (1),

$$
d V_{p}=V_{s}\left(\frac{R_{o}}{\left(Z_{p}+R_{o}\right)^{2}}\right) \cdot d Z_{p}
$$

and dividing both sides of Eq. (2) by the corresponding sides of Eq. (1) yield:

$$
\frac{d V_{p}}{V_{p}}=\left(\frac{R_{o}}{\left(Z_{p}+R_{o}\right)^{2}}\right) \cdot d Z_{p} /\left(\frac{Z_{p}}{Z_{p}+R_{o}}\right)=\frac{d Z_{p}}{Z_{p}}\left(\frac{R_{o}}{Z_{p}+R_{o}}\right)
$$

Using Eq. (1) and Eq. (3), we can relate the sensitivity of $V p$ and $Z p$, based on $V p$ and $V s$. 


$$
\begin{gathered}
\frac{d V_{p}}{V_{p}}=\frac{d Z_{p}}{Z_{p}}\left(1-\frac{V_{p}}{V_{s}}\right) \\
\frac{\% \Delta V_{p}}{\% \Delta Z_{p}}=\left(1-\frac{V_{p}}{V_{s}}\right)=\alpha
\end{gathered}
$$

Eq. (5) simply shows that the relative change in $V p$ is smaller than the relative change in the impedance magnitude $(Z p)$ by the factor of $\alpha$, which is always smaller than unity. For example, if $\alpha=0.5,10 \%$ change in the impedance would cause only $5 \%$ change in the measured Peak $V p$.

\section{S2.2. Current sensitivity}

Based on Fig. S3, we can express the amplifier's output current as Eq. (6).

$$
I_{p}=V_{S}\left(\frac{1}{Z_{P}+R_{o}}\right)
$$

Similar to Eqs. (2,3), we can drive the Eq. (7-9) to compare the sensitivities of Ip and $Z p$.

$$
\begin{gathered}
\frac{d I_{p}}{I_{p}}=\left(\frac{-d Z_{p}}{\left(Z_{p}+R_{o}\right)^{2}}\right) /\left(\frac{1}{Z_{p}+R_{o}}\right)=\frac{-d Z_{p}}{Z_{p}}\left(\frac{Z_{p}}{Z_{p}+R_{o}}\right) \\
\frac{d I_{p}}{I_{p}}=\frac{d Z_{p}}{Z_{p}}\left(\frac{-V_{p}}{V_{s}}\right) \\
\frac{\% \Delta I_{p}}{\% \Delta Z_{p}}=\left(-\frac{V_{p}}{V_{s}}\right)=\beta
\end{gathered}
$$

Eq. (9) shows that the maximum sensitivity in the $I p$ measurement happens when $V p$ is maximized. In other words, it means $R o$ needs to be as small as possible. Both sensitivity and $V p$ magnitude can be high together, which is entirely in contrast to what Peak detectors offer.

\section{S3. AC Current Measurement circuit - an example}

Measuring the amplifier's output AC current is not a straightforward task, particularly in the Megahertz frequencies. The well-known standard approach uses a trans-impedance amplifier with a test resistor in the feedback to convert the current signal to voltage signal, followed by a post-amplifier and finally a peak detector (Fig. S4). If one aims to implement such a circuit, one right choice for the trans-impedance amplifier is OP656 (Texas Instruments, TX), which ensures both the wide bandwidth and extremely low bias current ( 1 pico-ampere). Meanwhile, a variable gain amplifier like VCA824 (Texas Instruments, TX) would adjust the following Peak detector's input amplitude. Apart from the complexity of implementation, the nonlinearities in the gain of post-amplifiers and the peak detector would degrade measurement accuracy. 


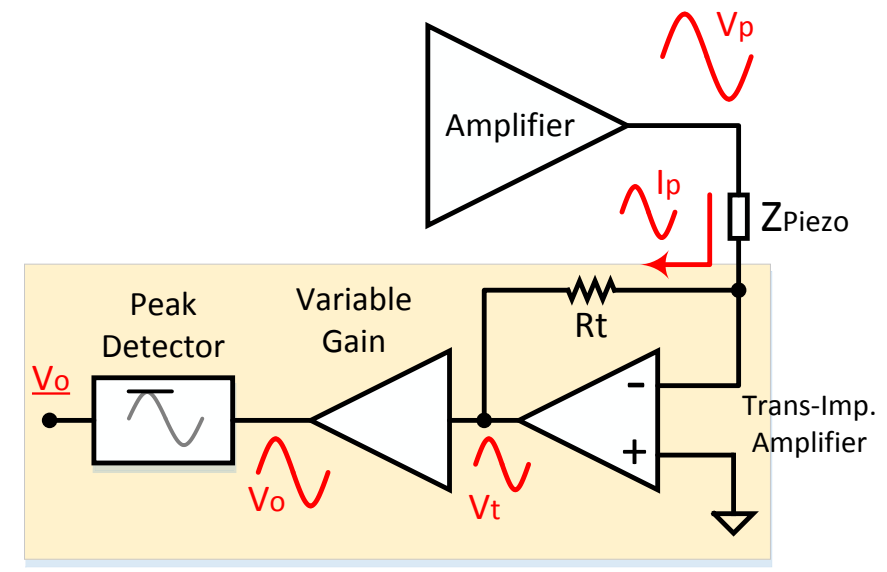

Fig. S4 An example for the implementation of AC current measurement unit

Several criteria should be taken into account when selecting the right components for the implementation of a custom amplifier.

1) The amplifier needs to be able to generate an output swing as large as $20 \mathrm{Vpp}$. It requires a dual power supply with symmetrical voltage levels of at least $\pm 12 \mathrm{~V}$ or preferably $\pm 15 \mathrm{~V}$.

2) At the resonance frequency of a piezoelectric transducer, the impedance magnitude reaches a minimum value while becoming more resistive than reactive. A smaller impedance value leads to a higher Ac current drawn from the amplifier. For the specific Piezo type used in our acoustic trapping system, the piezo impedance is around $70 \Omega$. Therefore, for a stimulation voltage of $20 \mathrm{Vpp}(\mathrm{Vp}=10 \mathrm{~V})$, the $\mathrm{AC}$ current has a peak amplitude of approximately $140 \mathrm{~mA}$ that should be handled easily by the amplifier system.

3) The amplifier's large-signal bandwidth needs to be high enough to provide a flat frequency response up to $9-10 \mathrm{MHz}$ for high voltage output.

4) The high output current capability would also necessitate precise control of the amplifier's power dissipation. The total power dissipation (PD) can be expressed as the difference between the amount of power from the power supply (through $V c c$ and $V e e$ terminals) into the amplifier and the delivered power to the impedance load $\left(P_{\text {Load }}\right)$ as Eq. (10).

$$
P_{D}=P_{\text {supply }}-P_{\text {Load }}=2 V_{c c}\left(I_{S 0}+\frac{I_{P}}{\pi}\right)-\frac{1}{2} V_{p} I_{p} \cos \theta
$$

Where $I_{S 0}$ is the DC component of supply current known as quiescent current and $I p$ is the amplitude of output AC current. In the second term, the parameter $\theta$ is the phase shift between the AC voltage and $A C$ current at the output. For a capacitive load, $\theta$ is around 90 degrees in frequencies away from the resonance point. However, this angle for a resistive load (at resonance) is close to 0.

By substituting the working parameters of our experiments $(V c c=15, I p=140 \mathrm{~mA}, V p=10 \mathrm{~V}$ and $\theta=0)$ into Eq. (11), a simplified expression for calculation of dissipated power is obtained: 


$$
P_{D}=2 V_{c c}\left(I_{S 0}+\frac{I_{P}}{\pi}-\frac{V_{p} I_{p}}{4 V c c} \underset{\cos 0}{1}\right) \cong 30^{V}\left(I_{S 0}+21^{m A}\right)
$$

Eq. (11) reveals that the DC quiescent current is also a critical factor in minimizing the power dissipation. However, apart from the Dc dissipation, the small impedance of load would dissipate more than $\sim 600 \mathrm{~mW}$ $(30 * 21)$ heat inside the amplifier that only a few single amplifiers could tolerate.

Apex's amplifier products could be a promising candidate if one is interested in implementing the custom amplifier with a single amplifier module (i.e., Operational Amplifier or OP-Amp). PA107DP is undoubtedly the best when it comes to providing all criteria mentioned above. It can operate with high supply voltage levels (up to $\pm 100 \mathrm{~V}$ ), $1.5 \mathrm{~A}$ output current capability, and maximum dissipated heat of $62 \mathrm{~W}$ with Heatsink. However, the minimum gain by which this amplifier can work properly (with stability) is 20 . Knowing that its gain Bandwidth (GBW) product is $180 \mathrm{MHz}$, the amplifier bandwidth becomes $9 \mathrm{MHz}$. It means we would lose $50 \%$ of power at this frequency (equal to $70 \%$ of output voltage) close to the working frequency in our experiment ( 7.5 to $8 \mathrm{MHz}$ ). Therefore, the selected amplifier should be stable in low gain values so that a more generous portion of its bandwidth is suitable for large signal amplification. Among thousands of commercially available OP-amps in the market, we nominated three OP-amps: LM7171, LM7372, and THS4631, all from Texas Instrument, TX. Table S1 summarizes the relevant features of these amplifiers.

Table S1. Comparison of three wideband operational amplifiers as candidates for the output stage of the custom amplifier

\begin{tabular}{|c|c|c|c|}
\hline & THS4631 & LM7171 & LM7372 \\
\hline Minimum Gain (Av) & 2 & 2 & 2 \\
\hline Slew rate (V/ $\mu \mathrm{S})$ & 1000 & 4100 & 3000 \\
\hline $\begin{array}{c}\text { Gain Bandwidth } \\
\text { Product (MHz) }\end{array}$ & 210 & 200 & 120 \\
\hline Supply Current (mA) & 11.5 & 6.5 & 13 \\
\hline lout max (mA) & 98 & 118 & 150 \\
\hline Package & $8-S O$ & 8 -DIP & $16-$ SOIC \\
\hline PD max at 25C (W) & $2.3^{*}$ & 1.1 & $2.6^{*}$ \\
\hline \#Circuits in a package & 1 & 1 & 2 \\
\hline
\end{tabular}

* If soldered to PCB with copper planes

Despite having a good power dissipation tolerance, the other features of THS4631 OP-Amp are the worst among this group, including the highest supply current, lowest output current, and lowest slew rate. The other amplifier ICs (LM7171 and LM7372) are comparable in several features such as slew rate, Supply Current (per circuit), and Power dissipation (per circuit). LM7372 offers a higher output current, but LM7171 has more power bandwidth. Based on Eq. (11), the maximum power dissipation will be around $850 \mathrm{~mW}$ (assuming $I_{S 0}=6.5 \mathrm{~mA}$ ). Both OP-amp candidates promise to tolerate this dissipation power. Nevertheless, this is not the whole story. According to the LM7171 datasheet, for the load impedance as low as $100 \Omega$, the maximum achievable output amplitude is around $14 \mathrm{Vpp}$ in lower frequencies that ends 
up to $10 \mathrm{Vpp}$ at $10 \mathrm{MHz}$ only yields $~ 50 \mathrm{~mA}$ output current (Fig. S5a). In other words, the output current of a single amplifier cannot reach the nominal value stated in the datasheet (118 mA). The power bandwidth of the amplifier has also been reduced. However, if the load has higher resistance, such as 700 $\Omega$, the output voltage amplitude of $20 \mathrm{Vpp}$ with little distortion $(<1 \%)$ at $8 \mathrm{MHz}$ is possible (Fig. S5b).
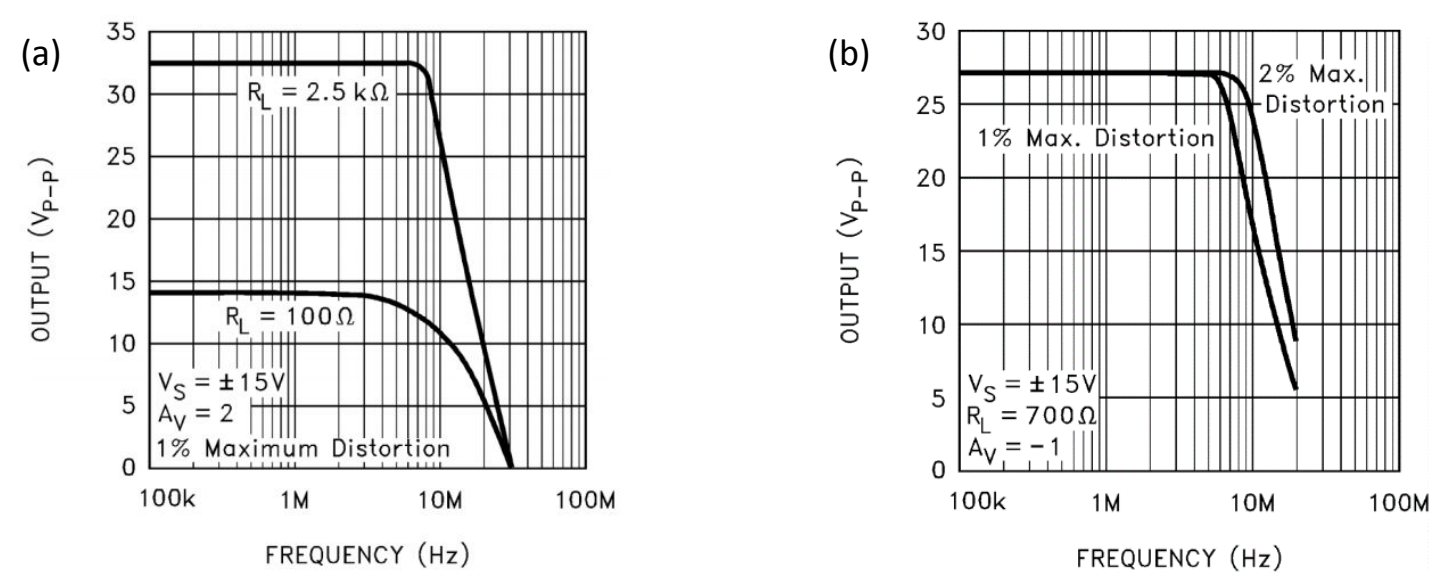

Fig. S5 Undistorted output swing for (a) RL $=100 \Omega$, and (b) $R L=700 \Omega$ in LM7171 operational amplifier

Hence, as a solution, an array of parallelized amplifiers is needed so that each amplifier IC (called: subamplifier module) contributes by providing a fraction of the overall output current. For this application, eight identical Op-amps (LM7171) were selected with the package type of DIP-8 (Dual in-line Package, eight pins) because they have better thermal resistance compared to smaller surface-mount counterparts (e.g., SOIC-8). Moreover, to have better thermal management, these eight ICs are divided into two groups of 4. Each group was directly attached to the corners of one rectangular thin Heatsink. The inputs and outputs of all sub-amplifiers are tied together in this parallel arrangement. All sub-amplifier modules have the same feedback structure (Gain $=2.5$ ) using precision resistors $(0.1 \%)$. As a result, all amplifier modules' outputs have the same phase and amplitude with high accuracy.

Nevertheless, the output signals are not identical due to the intrinsic tolerances in the fabrication of ICs and resistors. Therefore, a small resistor ( $33 \Omega$ ) at each amplifier's output provides isolation and prevents the over-current issue. These small protection resistors make an effective resistance of $4 \Omega(=33 / 8)$ at the whole amplifier system's output.

The non-inverting feedback in each sub-amplifier is $A v=2.5$, preserving the excellent power bandwidth of the LM7171 amplifiers. Each sub-amplifier is responsible for providing 18 mA (=140/8) of maximum output current around the resonance frequency. Following Eq. (9), this AC current results in a dissipated power of $\sim 275 \mathrm{~mW}$ inside each IC that does not raise their body temperature significantly. It is worth mentioning that using the heatsinks was not strictly necessary, but they prevent the ICs from becoming hot.

\section{S4. Current-meter unit}


A custom amplifier allows full access to all internal terminals/nodes of the system, including the supply pins. The maximum averaged current $\left(\overline{I_{S}} \max \right)$ into our custom amplifier is around $100 \mathrm{~mA}$, according to Eq. (12).

$$
\overline{I_{S}} \max =8 \times I_{S 0}+\frac{I_{P}}{\pi} \cong 8 \times 6.5^{m A}+\frac{140^{m A}}{\pi} \cong 100^{m A}
$$

To measure the averaged current $\left(\overline{I_{S}}\right)$, a small test resistor $(R T=0.25 \Omega)$ was added in series within positive supply line to produce a voltage equivalent $\left(V_{R T}\right)$ of supply current. Hence, the maximum of this voltage drop would be around $\sim 25 \mathrm{mV}$ that is negligible compared to $V c c(+15 \mathrm{~V})$, thereby not affecting the amplifier's performance. Here, the easiest and more accurate way to measure $V_{R T}$ is using a high precision current-sense amplifier like MAX44284F (Maxim Integrated, CA). This IC amplifies the input differential voltage with a fixed gain of 50 . Very low input bias current (max: $80 \mathrm{nA}$ ) ensures no wasted/leaked current during current to voltage conversion. Moreover, this IC can reject the common-mode DC voltage on its input terminals $(+15 \mathrm{~V})$ operating with only a lower voltage $(+5 \mathrm{~V})$ single supply. The Megahertz ripple on the supply current is far beyond this amplifier's sensible bandwidth ( $3 \mathrm{kHz}$ ). Nevertheless, a low-pass filter $\left(f_{3 d b}=\sim 1 \mathrm{KHz}\right)$ has been added at the input terminals, mainly to suppress the transient noise/interferences. The current-meter unit output is a $D C$ voltage ranging from $\sim 0.65 \mathrm{~V}$ to $1.2 \mathrm{~V}$, representing the amplifier's momentary averaged supply current.

\section{S5. Details on Resonance Frequency Tracking}

Finding the resonance frequency of the piezoelectric transducer mainly includes a single preliminary frequency sweep throughout a wide frequency range $\left(f_{1}\right.$ to $\left.f_{2}\right)$ and a sequence of continuous local (narrow range) sweeps around the latest found resonance frequency. 


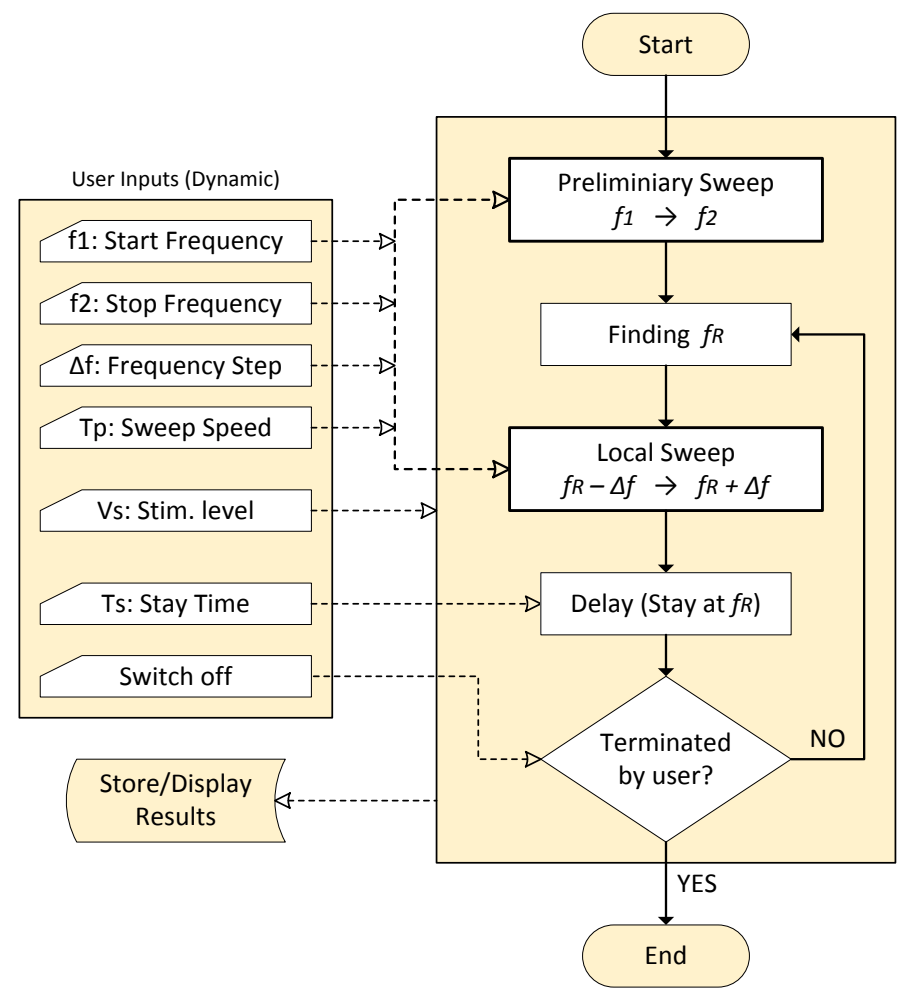

Fig. $\mathbf{S 6}$ The simplified flowchart of the instruction for real-time tracking of the resonance frequency

Fig. S6 Illustrates the basics of this procedure. The left box inside this figure shows sweep parameters that the user can change during the frequency tracking operations. The dashed arrows indicate the relationships among the sweep parameters and the instructions.

Fig. S7 shows the variation of output current and voltage amplitude during the Preliminary Sweep (Zoomed View) for both Voltage-based (a) and Current-based approaches (b). In the Voltage-based method, output amplitude drops significantly (below $10 \mathrm{Vpp}$ ) around the resonance frequency, causing less effective Acoustic trapping. On the other hand, the amplitude drop in the Current-based method is substantially low (18 Vpp), and the maximum current determines the resonance frequency.
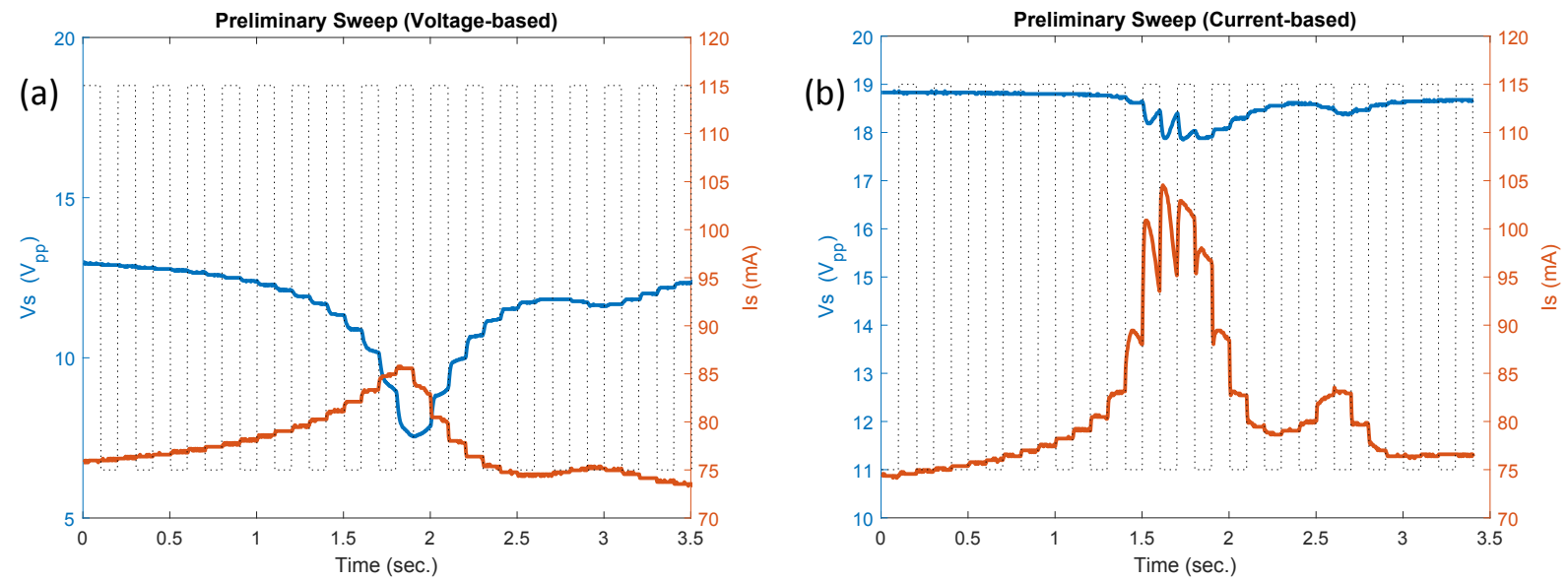

Fig. S7 The zoomed view of detailed preliminary sweeps around the resonance frequency for two approaches: (a) 
Voltage-based and (b) Current-based. The frequency step was $2 \mathrm{kHz}$ with a stay time of $0.1 \mathrm{sec}$ at each frequency point. The blue plots are Output amplitudes $(V s)$ against the left $y$-axis and, the red plots are Supply currents (Is) against the right $y$-axis. The Up-Down dotted lines indicate when the frequency hopped to the next frequency point.

\section{S6. Multiplexed Acoustic Trapping system}

In several microfluidic sample preparation applications, there is a need for local control of the resonance frequency of multiple channels in parallel, depending on the sample being manipulated. After a sexual assault, for instance, victims undergo a thorough forensic examination of their bodies. During this examination, four evidentiary swabs are collected from the victim, a vaginal swab, a vaginal-cervical swab, a perianal swab, and an external genitalia swab. All of these swabs have to undergo the time-consuming process of differential extraction. Differential extraction (DE) is the process by which forensic scientists separate sperm cells (from the perpetrator) and epithelial cells (from the victim) in order to produce a genetic profile of the perpetrator. This process requires a trained technician to first selectively lyse the female epithelial cells before a series of centrifugation and washing steps are conducted to obtain a purified sperm pellet that can then go on for downstream extraction, nucleic acid amplification, and capillary electrophoretic separation to produce a genetic profile that can be used to identify the perpetrator. The development of the SONIC-DE system allows for the elimination of the time-consuming steps of centrifuging and washing. When tested by real forensic scientists, it was considered a promising replacement for their current workflow. However, as the current system is only able to process one swab at a time, it was brought to our attention how valuable it would be to process multiple samples simultaneously. Having an instrument that is capable of processing four samples simultaneously would work effortlessly with the current workflow, as analysts are commonly working on a single case at a time to prevent any contamination.

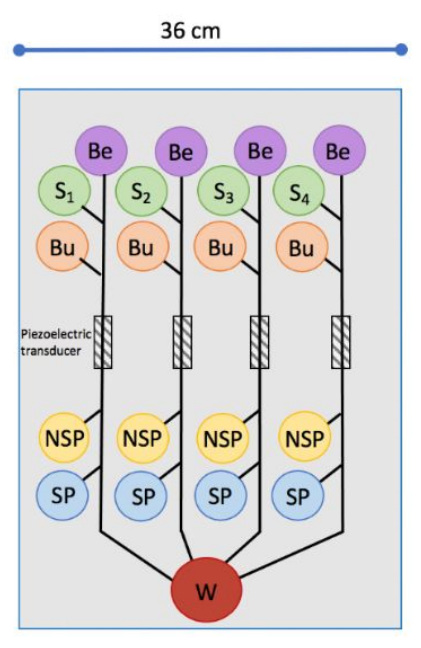

(a)

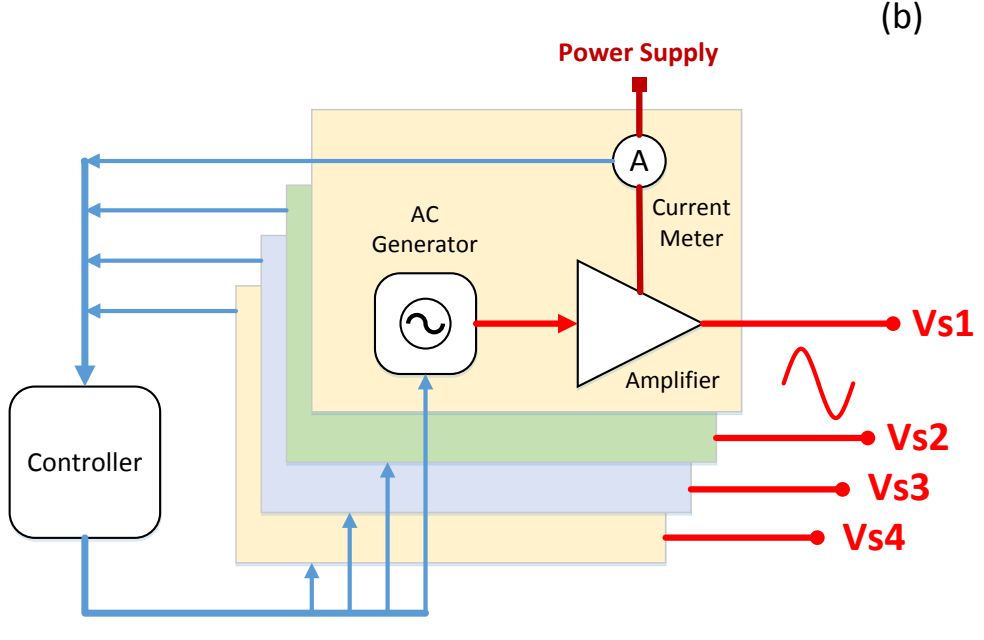

Fig. S8 The schematic of (a) quad-channel acoustic trapping chip and (b) Four-channel Acoustic trapping controller board 
This multi-channel acoustofluidic chip (Fig. S8a) and instrument capable of video monitoring the acoustic trapping in each channel is being completed. Additionally, the currently implemented system will be expanded to include four independent modules. Each includes a signal stimulation unit and current measurement unit that will independently control each trapping site, applying the resonant frequency based on real-time current measurements (Fig. S8b). We aim to develop this system and validate it for various mock sexual assault swabs before testing the prototype instrument in a real forensic lab. Some examples are shown in Fig. $\mathbf{S 9}$

(a)

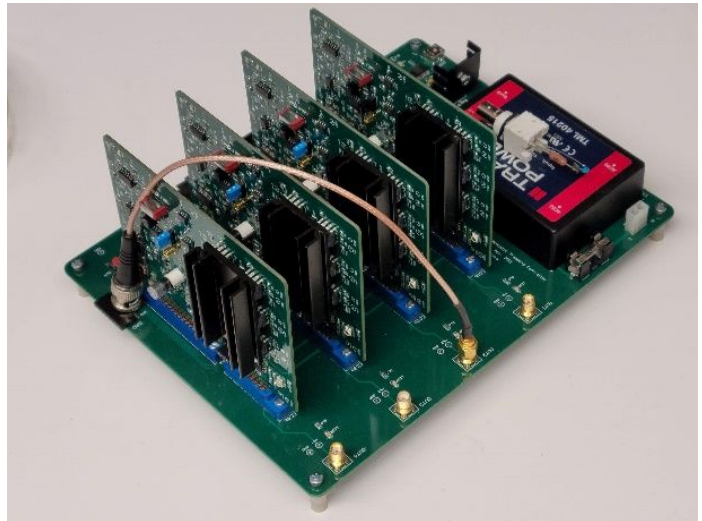

(b)

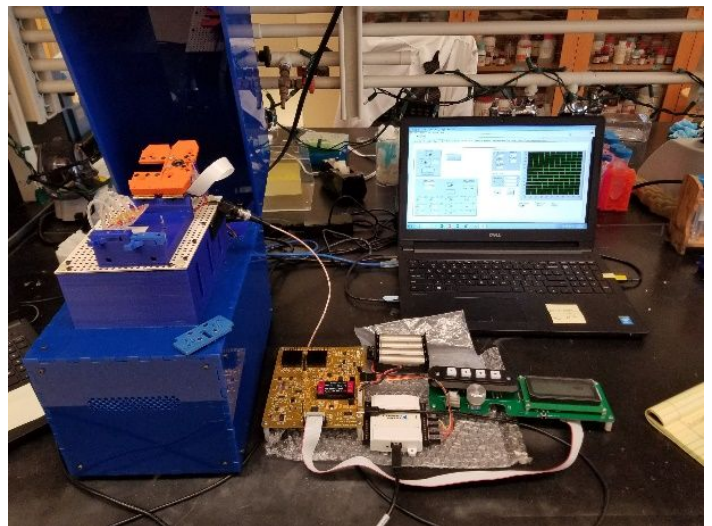

Fig. S9: (a) Control board for multichannel (4-channel) resonant frequency measurement and control with portable power options; (b) integrated within the broader acoustic sample manipulation system. 